\title{
Evaluate the Effectiveness of Cognitive-Behavioral Therapy on Emotion Regulation and Cognitive Processing in Patients with MDD
}

\author{
Seyedeh Mehri Katoorani ${ }^{1}$, Hamze Ahmadian ${ }^{2}$, Narges Shams Alizadeh ${ }^{3}$
}

${ }^{1}$ Corresponding Author, MA in Clinical Psychology, Department of Psychology, Islamic Azad University, Sanandaj, Iran.

${ }^{2}$ Assistant Professor of Psychology, Islamic Azad University, Sanandaj, Iran. Ahmadian2012@gmail.com

${ }^{3}$ Assistant Professor of Kurdistan University of Medical Sciences.

\section{ARTICLE INFO}

Keywords:

Cognitive-Behavioral

Therapy, emotion

regulation, Cognitive

Processing, Major

Depressive Disorder

\begin{abstract}
This study aimed to evaluate the effectiveness of CognitiveBehavioral Therapy on emotion regulation and Cognitive Processing in patients with MDD. For this purpose, 30 patients with major depressive disorder in Sanandaj city were selected by convenience sampling method and replaced in two 15-subject groups. At first, both groups responded to emotional regulation and cognitive processing questionnaires. Then, for the experimental group, 8 sessions of the cognitive behavioral therapy program were implemented in a group manner, but no intervention was performed for the control group. After intervention, the data of emotional regulation and cognitive processing questionnaires were analyzed from the patients in two groups in the pretest and posttest phases using the ANOVA test. The findings showed that cognitive behavioral therapy program has a significant effect on improving emotional regulation and cognitive processing of patients with major depressive disorder.
\end{abstract}

\section{Introduction}

The use of strategies of emotional cognitive adjustment compatible in stressful situations and negative emotions is an important factor in promoting physical and mental health. Therefore, the proper and desirable use of cognitive behavioral strategies in emotional regulation is effective in reducing depression and will cause the cognitive reconstruction of the problem and, as a result, mental health promotion. One of the important issues in contemporary psychology is the discussion of how emotional disturbances, such as depression, affect cognitive functions. Depression is one of the problems that affects a significant percentage of the community and includes a series of pathological syndromes such as depressed mood, loss of interest, sleep disturbance, lack of energy, loss of appetite and suicidal thoughts. Major depressive disorder is one of the most common psychiatric disorders (Avenevoli, Shelli, Swendsen, 2015). Studies by Sullivan and Payne (2007), Airaksinen, Larsson \& Forselli (2005), Ghomri and Khoshnam (2013), Larijani, Mohammad Khani, Hassni, Sepah Mansour and Mahmoudi (2014) have shown that patients with major depressive disorder and anxiety suffers from cognitive impairment. Depression is the second most common psychological disorder affecting more than 200 million people in the world, and new data from Hassin and colleagues show that

* Corresponding Author E-Mail Address: mkat.teach@gmail.com 
nearly 300 million people in the world are depressed. Emotional, social and economic burden of depression for patients, family and society on the one hand, and the estimating annual outbreak of 2.9 to $12.6 \%$ of the general population (Hasin, D. S., Goodwin, R. D., Stinson, F. $\mathrm{S}, 2005$ ), has remained depression in the area of mental health issues as one of the major pillars of research.

According to international statistics, $15 \%$ of people between 15 and 74 years old who refer to doctors have symptoms of depression. Many depressed people have difficulty in thinking, concentrating, or making decisions. They may easily be distracted or have a memory problem. In most cases, it may be existed the thoughts about death, thoughts about suicide or attempt to suicide. The scope of these thoughts is from thinking to suicide to act on it. The abundance, intensity and fatalities of these thoughts also fluctuate (Kaplan, Saduk; translation of Reza'i, 2014).

Research by Joorman (2010) and Ehring (2010) shows that patients with major depressive disorder are not able to inhibit negative information, and, on the other hand, they increase levels of repetitive negative thinking such as rumination, which may interfere in applying adaptive strategies of emotional cognitive regulation, such as reassessment and causes continuation of depression disorder.

Because emotional cognitive regulation strategies allocate an important part of every person's life, it's not surprising that confusion in excitement and regulation can cause mental harm (Cisler, J. M., \& Olatunji, B. O., 2012). Several studies have examined the effectiveness of cognitive behavioral therapy in mental disorders (Thomas et al., 2012; Stace et al., 2008; Clarke, 1999; Ahmadkhaniha, 2011; Hosseini et al., 2004). Therefore, with regard to the mentioned issues, this study attempts to answer the question of whether the effectiveness of cognitive behavioral therapy affects emotional regulation and cognitive processing of patients with major depressive disorder.

One of the most important issues in depression is the issue of cognitive emotional regular (CER) and regulation of emotion in these patients which is one of the important areas of behavioral actions that are interdependent with depression and play an essential role in empowerment and adaptation of people with environmental requirements (Klingman, 2009). For the two components, control emotions, coping with the stress from training program of life skills allocates to the strategies of cognitive emotion regular. Cognitive emotional regular strategies are very broad, inclusive which contains a broad range of mental, physiological and behavioral processes (Garnefski $\mathrm{N}$ et al., 2007). The basic point is that emotions are an important part of our components of life that affect our lives (Sarp and Tosun, 2011). The data obtained from Berking, M., Ebert, D., Cuijpers (2013) indicates the role of maladaptive strategies of emotional cognitive regular in the continuation of depression disorder. These data suggest that the correction of the maladaptive strategies of emotional cognitive regular can reduce symptoms of depression.

Different theories have been proposed in explaining the causes of depression. Therapeutic methods have also been developed based on existing theories. Depression is examined from biological, psychoanalytic, cognitive-behavioral points of view and system theories. According to the etiology of this disorder, various therapeutic approaches have been proposed. One of these approaches is cognitive behavioral therapy that has been proposed since the 1970s for the treatment of a variety of depressed patients and its usefulness has been confirmed in some studies (Oei and Dingle, 2007).

Cognitive-Behavioral Therapy is a structured, purposeful psychotherapy method focused on the present time that uses cognitive and behavioral strategies to reduce symptoms by targeting defective cognitive constructs and maintenance processes of mental disorders. (Grant \& Beck, 2010). 
Because depression is very stressful for the patient and the family and creates a lot of costs for drug treatment, disability or hospitalization, therefore, in order to plan appropriate psychotherapy measures, promote psychological health services and create related policies in society, it needs to psychotherapy with appropriate treatments such as cognitive-behavioral therapy. Although drug therapy is the stabilized drugs in the treatment of major depressive disorder but treatment results are dependent on other side effects. In a meta-analysis study, comparing cognitive-behavioral therapy with other therapies has shown that despite the benefit of drug therapy because of lower cost, response to treatment and its faster effect on physical and mood symptoms, cognitive-behavioral therapy effects deeply on the cognitive structure and the patient's deep cognitive and emotional processing (Wempold et al., 2002). Most studies have reported the combination of drug therapy with psychotherapy (American Psychiatric Association, 2000).

One of the psychotherapy approaches is cognitive-behavioral therapy. The term cognitive behavioral therapy was first used in the medieval scientific literature of the 1970s, and the empirical foundations of cognitive-behavioral approaches to psychological issues returns at the beginning of this century. Aaron in the book of Theory of Cognition and Emotion Disorders (1976) has stated the principles of his theory. In his opinion, cognitive aspects such as negative perceptions of the person from the outside, low self-esteem, and his general disappointment over the future, rather than the result of a depressed feeling involve such emotions. A depressed patient essentially considers him an unlucky person. Anything to do is nothing but a failure and loss. Therefore, he refuses to accept any purpose or action for any constructive work and excludes the whole future from any pleasure or success (Shamloo, 2004).

Based on this view, the experiences of individuals lead to the formation of assumptions or schemas about themselves and the world, and these assumed schemas affect perceptual organization and control of behavior evaluation and lead to depression (Robertson, 2010; Wampold et al. 2002). Cognitive-Behavioral Therapy is essentially an approach that its using has been considered for treating a wide range of psychiatric disorders and numerous theoretical and experimental supports. Cognitive-behavioral therapy can be used both in individual treatment situations and in group treatment situations and achieved good results (Khodayari Fard, 2010).

The results of the research by Jabbar, Mahmoudi and Paydar (2015), showed that cognitivebehavioral intervention can be used as one of the effective therapeutic methods in students' emotional-behavioral problems. The findings of Bagherinia , Javadi and Nurani (2015), showed that cognitive-behavioral and mind-focused therapy is effective in improving the emotional regulation in patients with major depression. Zare Bahram Abadi, Nazari (2014), in a research that showed that cognitive therapy group significantly reduced depression and increased self-efficacy in the experimental group compared with the control group. Solati Dehkordi and Mobashari's research (2012) also showed that cognitive-behavioral group therapy and family psychological education along with drug therapy rather than purely drug therapy can be more effective in improving symptoms of depressed patients.

Foreign research has also confirmed the educational effect of emotional regulation skills on depression treatment, among the researches; it can be referred to the research of Ahrat et al. (2014), which indicated that the hypothesis of training of emotional regulation skills has been effective on reducing the symptoms of depression in the experimental group. Thomas LJ, Abel A, Ridgway N, Peters T, Kessler D, Hollinghurst S (2012) reported that symptoms of depression in the cognitive behavioral group were significantly reduced than those receiving medication alone. O'Mahena, \& et al (2012), also in a research classified cognitive-behavioral therapies for early depression, titled What do women want? The results of this study indicated the effectiveness of cognitive-behavioral therapy on depression in women with depression. 
Considering the above issues and the questions posed and based on existing theoretical researches, one of the objectives of this study is to determine the effectiveness of cognitive behavioral therapy on cognitive processing of patients with depression among adults in Sanandaj. Another goal is to determine the effectiveness of cognitive behavioral therapy on cognitive emotion regular among individuals. Based on these goals, two hypotheses were formed:

1- Cognitive behavioral therapy is effective on cognitive processing in patients with major depressive disorder.

2- Cognitive behavioral therapy is effective on cognitive emotion regular in patients with major depressive disorder.

\section{Method}

The present research is applied objectively and in terms of method is semi-experimental from pre-test and post-test type with the control group. Considering that in this study, it was tied to be investigated the effectiveness of cognitive behavioral therapy on cognitive emotion regular and cognitive processing of patients with major depressive disorder in two experimental and control groups. Therefore, the mentioned method is a suitable method. The statistical population of this study was all adult males and females with major depressive disorder referring to Sanandaj psychiatric and psychology and counseling centers in the first half of 2017. Using convenience sampling method, 30 patients with psychiatric diagnosis of major depressive disorder were selected as a statistical sample in the first half of 2017 and randomly replaced into two groups of 15 experimental and control groups. The research tools included two standard questionnaires:

Emotion regulation Questionnaire prepared by Grass \& John (2003), the above scale consists of 10 items that have two subscales of reassessment (6 items) and suppression (4 items). Responses are based on Likert scale (7-degree), totally opposite (1) to totally agree (7). Cronbach's alpha coefficient for re-assessment was 0.79 and for suppression was 0.73 and retest validity after 3 months for the whole scale is reported 0.69 (Grass \& John, 2003). The internal consistency coefficient of this scale was reported for state employees and Catholic students at the University of Milan to re-assess with a positive emotional scale (0.24) and negative emotions (-0.14), and suppression with positive emotions (-0.15) and negative emotions (0.04) (Balzuritti, John and Grass, 2010). The Persian version of Grass and John's emotion regulation questionnaire in Iranian culture by Qasempour, Il Beigi and Hasanzadeh (2012) has been standardized.

Cognitive Processing of Information Questionnaire This 24-questions scale is made by Schouwenburg, Schlder (1996), and has 3 critical reading scales, basic texture and structure. The total score of these 3 subscales is the total score of the cognitive processing of information, which is scored as a 5-point Likert scale. The reliability of the test has been reported from 0.75 to 0.81 and its validity 0.79 (Qasempour, 2011). Qasempour (2011) also confirmed the reliability and validity of the test in his research.

The reliability coefficient in the present study is 0.79 . In this research, the validity of the scale is reported based on internal consistency (Cronbach's alpha coefficient from 0.60 to 0.81 ) and validity of the questionnaire through main component analysis using varimax rotation, correlation between two subscales $(r=0.13)$ and desirable validity.

In this research, in order to answer the questions to analyze the collected data, descriptive statistics (mean and standard deviation, percentage frequency, score range) is used and in the level of inferential statistics after observing the hypotheses, the statistical test and covariance analysis is used. 


\section{Findings}

Before analyzing the research data, the underlying assumptions of the analysis were studied. The most important of these assumptions is the assumption of normality. Kolmogorov Smirnov (K-S) was used to investigate the normality of data of distribution. The KolmogorovSmirnov test for all variables was calculated, with the results as follows:

Table 1: The result of the normal test of cognitive emotion regular and cognitive processing variables

\begin{tabular}{|l|c|c|c|c|}
\hline \multicolumn{2}{|c|}{ Variables } & N & Sig & Error value \\
\hline Cognitive emotion regulation & Pretest & 15 & 0.235 & 0.05 \\
\cline { 2 - 5 } & Posttest & 15 & 0.269 & 0.05 \\
\hline \multirow{2}{*}{ Cognitive processing } & Pretest & 15 & 0.968 & 0.05 \\
\cline { 2 - 5 } & Posttest & 15 & 0.388 & 0.05 \\
\hline
\end{tabular}

The results of the table showed that the Kolmogorov-Smirnov test for cognitive emotion regulation and cognitive processing variables is $(0.79,0.49)$ in pretest, respectively, as well as the Kolmogorov-Smirnov value for cognitive emotion regular variables and cognitive processing in the post-test is $(1.05,1.90)$, respectively. The significant level of each variable in the two groups is more than the error value (0.05) in the pretest and posttest groups. Therefore, it can be said that the distribution of data is normal in the two groups.

Table 2: Mean scores and standard deviation of subjects in emotion regulation and information processing variables in both experimental and control groups

\begin{tabular}{|l|c|c|c|c|c|c|}
\hline \multirow{2}{*}{ Variable } & \multirow{2}{*}{ Group } & \multirow{2}{*}{ Number } & \multicolumn{2}{c|}{ Pretest } & \multicolumn{2}{c|}{ Posttest } \\
\cline { 4 - 7 } & & & Mean & SD & Mean & SD \\
\hline \multirow{2}{*}{ Cognitive emotion regular } & Experimental & 15 & 38.73 & 2.81 & 40.86 & 2.55 \\
\cline { 2 - 7 } & Control & 15 & 38.06 & 3.26 & 38.53 & 3.18 \\
\hline \multirow{2}{*}{ Cognitive processing } & Experimental & 15 & 0.0050 & 3.11 & 52.00 & 3.58 \\
\cline { 2 - 7 } & Control & 15 & 0.2048 & 2.85 & 49.00 & 2.23 \\
\hline
\end{tabular}

The results of Table 2 show that the mean of cognitive emotion regular and cognitive processing after intervention in the experimental group have increased to some extent than control group, regarding the standard deviation scores in the two groups before and after the intervention, in all variables, variables are homogeneous in terms of standard deviation scores.

First hypothesis: Cognitive behavioral therapy has an impact on cognitive emotion regulation of patients with major depressive disorder.

Table 3: co-linearity of data of cognitive emotion disorder

\begin{tabular}{|c|c|c|c|c|}
\hline Model & $\begin{array}{c}\text { Coefficient of } \\
\text { determination (R2) }\end{array}$ & T value & Sig & VIF \\
\hline Emotion regulation posttest & 0.034 & 1.41 & 0.167 & 1.000 \\
\hline
\end{tabular}

In the above table, given the value $\mathrm{T}$ and non-significant, it can be said that the data are linear. But given that vif is equal to (1/00), which is smaller than the number two, it can be said that the linearity of data is in a small amount. The results of the covariance analysis and multivariate analysis of variance analysis of the data were analyzed. The results of the statistical presumptions showed that the prerequisite for the equation of variances (using Levine test) 
with respect to the $\mathrm{F}=1.45$ and the greater significance level of Levine test than $0.05 \%$ can be said that the post-test homogeneity is considered, and the test of similarity of line slope of regression is the same for the experimental and control groups. Interaction between the group and the cognitive emotion regular pre-test is not significant. In other words, the data supports the homogeneity hypothesis of the regression slopes $(\mathrm{P}=060, \mathrm{~F}=3.13)$.

Table 4: One-variable covariance test for the effect of cognitive behavioral therapy on cognitive emotion regular in patients with depression

\begin{tabular}{|l|c|c|c|c|c|c|c|}
\hline \multicolumn{1}{|c|}{ Source of variants } & $\begin{array}{c}\text { Sum of } \\
\text { squares }\end{array}$ & df & $\begin{array}{c}\text { Mean of } \\
\text { squares }\end{array}$ & F value & Sig & $\begin{array}{c}\text { Statistical } \\
\text { power }\end{array}$ & $\begin{array}{c}\text { Chi } \\
\text { Eta }\end{array}$ \\
\hline Constant value & 169.88 & 1 & 169.88 & 20.79 & 0.000 & 0.435 & 0.993 \\
\hline Cognitive emotion regular & 12.91 & 1 & 12.91 & 1.58 & 0.219 & 0.055 & 0.228 \\
\hline Groups & 34.35 & 1 & 34.35 & 4.32 & 0.047 & 0.138 & 0.518 \\
\hline Error & 220.55 & 27 & 8.16 & & & & \\
\hline Total & 47557.00 & 30 & & & & & \\
\hline
\end{tabular}

As shown in Table 4, the F-value test for counteracting the effect of cognitive behavioral therapy on cognitive emotion regular in patients with depression is 4.32 and its significance level is ( $\mathrm{p}<0.05$ (0.047). Therefore, with a 5\% error probability, it can be said that the counteracting of cognitive behavioral therapy has a significant effect on cognitive emotion regular of patients with depression. ETA squared in these analyzes means how much the changes in post-test scores results from changes in group membership (control or experiment group), which results 0.19 of post-test variations.

Table 5: Average modulation of groups in cognitive emotion regular

\begin{tabular}{|l|l|c|c|}
\hline Variables & \multicolumn{1}{|c|}{ Group } & Mean & SD \\
\hline \multirow{2}{*}{ Cognitive emotion regular } & Experimental & 40.79 & 0.74 \\
\cline { 2 - 4 } & Control & 38.60 & 0.74 \\
\hline
\end{tabular}

In the table below, the adjusted averages of posttest of cognitive emotion regular indicate that the mean of the control group is lower than the experimental group. It also shows the effectiveness of training on the experimental group, and this difference according to the moderated averages is for the benefit of the training group.

Second hypothesis: The cognitive behavioral therapy affects the cognitive processing in patients with major depressive disorder.

Table 6: linearity of cognitive processing data

\begin{tabular}{|c|l|l|l|l|}
\hline Model & $\begin{array}{c}\text { Coefficient of } \\
\text { determination (R2) }\end{array}$ & T value & Sig & VIF \\
\hline Cognitive processing & 0.027 & 0.88 & 0.386 & 1.000 \\
\hline
\end{tabular}

In the co-linearity, the value of the determination coefficient (R2) is usually high and the significant amount (t) is low. With regard to the inflation factor of variance (VIF), the more variance (VIF) is greater than 2; the co-linearity is greater. In the above table, given the T value and the non-significant $\mathrm{T}$, it can be said that the data are linear. The results of the covariance analysis and multivariate analysis of variance of the data were analyzed. The results of the statistical presumptions showed that the prerequisite for the equation of variances (using 
Levine test) with respect to the value of $\mathrm{F}=1.96$ and greater than the significance level of the Levine test of $0.05 \%$ can be said that the post-test homogeneity assumption is observed. And the test of similarity of regression for control and experimental groups is the same, I.e interaction between the group and the cognitive pre-test is not significant. In other words, the data supports the homogeneity hypothesis of the slopes of the regression $(\mathrm{P}=.059 \mathrm{~F}=3.15)$.

Table 7: One-variable covariance test for the effect of cognitive behavioral therapy on cognitive processing in patients with major depressive disorder

\begin{tabular}{|l|c|c|c|c|c|c|c|}
\hline Source of variants & Sum of squares & $\mathbf{d f}$ & $\begin{array}{c}\text { Mean of } \\
\text { squares }\end{array}$ & $\begin{array}{c}\text { F } \\
\text { value }\end{array}$ & Sig & $\begin{array}{c}\text { Statistica } \\
\text { 1 power }\end{array}$ & Chi Eta \\
\hline Constant value & 247.82 & 1 & 247.82 & 26.79 & 0.000 & 0.498 & 0.999 \\
\hline Cognitive processing & 0.25 & 1 & 0.25 & 0.028 & 0.869 & 0.001 & 0.053 \\
\hline Groups & 59.19 & 1 & 59.19 & 6.39 & $\mathrm{P}<0.018$ & 0.192 & 0.684 \\
\hline Error & 249.74 & 27 & 9.25 & & & & \\
\hline Total & 86725.00 & 30 & & & & & \\
\hline
\end{tabular}

As shown in this Table, the F-value test for counteracting the effect of cognitive behavioral therapy on cognitive emotion regular in patients with depression is 6.39 and its significance level is ( $p<0.05$ (0.018). Therefore, with a 5\% error probability, it can be said that the counteracting of cognitive behavioral therapy has a significant effect on cognitive emotion regular of patients with depression. ETA squared in these analyzes means how much the changes in post-test scores results from changes in group membership (control or experiment group), which results 0.15 of post-test variations.

Table 8: Average modulation of groups in cognitive processing

\begin{tabular}{|l|l|c|c|}
\hline Variables & Group & Mean & SD \\
\hline \multirow{2}{*}{ Cognitive processing } & Experimental & 51.97 & 0.80 \\
\cline { 2 - 4 } & Control & 49.02 & 0.80 \\
\hline
\end{tabular}

In the table below, we observe the adjusted post-test averages of cognitive processing. The effect of auxiliary random variables is statistically eliminated. Averages indicate that the average of the experimental group is lower than the control group. It also shows that there is a significant difference between the experimental group that was affected by the training and the control group that was not under any therapy plan. And this difference is due to moderated averages is for the benefit of the trained group. Therefore, it is concluded that cognitive behavioral therapy has an impact on cognitive processing in patients with major depressive regular

\section{Discussion and Conclusion}

The results of data analysis showed that there is a significant difference between interventions of cognitive behavioral therapy on emotion regulation and cognitive processing of patients with major depressive disorder.

There is a significant relationship between cognitive behavior therapy and cognitive processing in patients with major depressive disorder. i.e, cognitive behavioral therapy has an impact on cognitive processing.

The results of data analysis showed that cognitive behavioral therapy was effective on cognitive processing in patients with major depressive disorder. The results obtained for this hypothesis 
are consistent with researches of Weingartner, H (1993), Bazin et al. (1994), Austin (1999), Kush and Fleming (2000), Watkins et al (2000), Spring et al (2003), Hamar et al. (2003), Gallassi (2006), Oei and Dingle (2007), Gomri Gio, Bashrpour, Narimani (2008), Abolqasemi et al (2011), Pourmohammadi and Ghaderi (2015).

In analyzing the hypothesis, it can be said that the symptoms of mood disorders is divided into emotional, cognitive, physiological and behavioral domains. Emotionally, depressed, sad, and frustrated mood is the basic symptoms. Cognitively, declining concentration, memory loss, memory bias, mental retardation, and suicidal thoughts are the main symptoms. Physiologically, insomnia, oversleeping, increase or decrease appetite, and weight and impairments are the main symptom, and the field of behavior includes the slowdown of physical activities.

About $50 \%$ to $75 \%$ of depressed patients have cognitive impairment and impairment of information and memory processing, sometimes called false dementia of depression. These patients are often plaintiff about negligence and concentration (Kaplan and Saduk, 2003). Cognitive deficits in depressive disorder are that, on the one hand, several reports claim that depressed patients show incompetence in situations where they need to alert retrieve of an event studied (explicit memory tasks such as free recall). While other studies have shown that function in implicit memory tasks in people suffering from depression remains intact. An interpretation to distinguish between explicit memory deficits and intact implicit memory function is that depressed patients are defective in the ability to use controlled retrieval strategies, while these patients have no problems with the use of automated processes (Hartlag et al., 1993).

Thus, the information on the memory of depressed patients sometimes emphasizes the existence of deficiencies in explicit memory, and sometimes emphasizes the existence of deficiencies in implicit memory. On the one hand, people such as Wayne Gartner et al. (1993) and Bazin et al. (1994) emphasize only on disorder in controlled processing, on the other hand, researchers such as Austin et al. (1999) emphasize the damage in both types of memory in depressed patients.

There is a significant relationship between the concepts of cognitive behavioral therapy and the emotion regulation of patients with major depressive disorder. i.e, cognitive behavioral therapy has had an effect on emotion regulation. In other words, it causes emotion regulation of patients with depression.

In analyzing this hypothesis, it can be said that cognitive behavioral therapy has been effective on emotion regulation of patients with major depressive disorder. The results are consistent with results of Berking, M., Ebert, D., Cuijpers, P., \& Hofmann (2013), Goldin, P. R., \& Gross (2010), Chambers et al. (2009), Anderson, Nicole D., Lau, Mark A., Segal, Zindel V., \& Bishop (2007), Ghasemzadeh Nasaji et al. (2010)), Azargoon and Kajbaf (2010), Kiani, Ghasemi, Pourabbas (1392).

On the effectiveness of cognitive-behavioral methods, we can point to the above-mentioned studies that using cognitive-emotional strategies compatible in stressful situations and negative emotions is an important factor in promoting physical and mental health. Therefore, proper and desirable use of cognitive behavioral strategies in emotional regulation is effective in reducing depression. And it causes the cognitive reconstruction of the problem and, as a result, improves mental health and since behavioral methodology challenges negative thoughts such as blaming self and replacing rational thoughts, including positive reappraisal, in this way, individuals are 
asked to emphasize on this issue and practice it, therefore, behavioral methodology can have a significant effect on the emotional regulation (Garnafski et al., 2001).

In the end, it is suggested:

Cognitive-behavioral therapy for patients with other psychological problems with regard to the effectiveness of this program should be implemented on patients with major depressive disorder. Materials tailored to families and mental health instructors should be prepared. Workshops for therapists for proper implementation and operating training programs should be hold. It is also suggested to other researchers to carry out qualitative research in order to identify the strengths and weaknesses of this method and provide appropriate training strategies in the field of cognitive-behavioral therapy.

\section{Resources}

Abolghasemi, Abbas; Ghomri Givi; Hossein; Bashrpour; Sajjad; Narimani, Mohammad (2015). Effectiveness of cognitive processing and holographic re-processing in reducing the psychological symptoms of traumatic students, Journal of Clinical Psychology, 3 (11), 5382.

Ahmadkhaniha, Hamid Reza; Gharaeipour, Manoochehr; Pennaghi, Leily (2011). The Effectiveness of Dependency Management and Cognitive-Behavioral Therapy in Opiate Dependence, 12 (1), 8-3.

Anderson, Nicole D., Lau, Mark A., Segal, Zindel V., \& Bishop, Scott, R. (2007). Mindfulness-based stress reduction and attentional control. Clinical Psychology \& Psychotherapy, 14 (6), 449-463. doi: 10.1002/cpp.5.

Austin, M. P, Mitchell, P.\& Wilhelm, K, (1999). Melancholic depression: a pattern offrontal cognitive impairment, Psychological Medicine, 29, 73-85.

Avenevoli, Shelli, Swendsen, Joel, He, Jian-Ping, Burstein, Marcy, \& Merikangas, Kathleen Ries. (2014).

Bagherinia, Hasan; Yamini, Mohammad; Javadi Elmi, Leila; Nourdi, Tayebeh (2015). Comparison of the Effectiveness of Cognitive-Behavioral Therapy and Mind-Based Therapy on Cognitive-Emotional Regulation in Major Depressive Disorder, Yasouj University of Medical Sciences. 20 (3) .210-219.

Bazin, N., Perruchet, D., Bonis, M., \& Feline, A. (1994). The Dissociation of Explicit and Implicit Memory in Depressed Patients. Journal of Psychological Medicine, 24, 239 - 245.

Berking, M., Ebert, D., Cuijpers, P., \& Hofmann, S. G. (2013). Emotion regulation skills training enhances the efficacy of inpatient cognitive behavioral therapy for major depressive disorder: a randomized controlled trial. Psychother Psychosom, 82(4), 234-245. Doi.

Chambers, Richard, Gullone, Eleonora, \& Allen, Ni cholas B. (2009). Mindful emotion regulation: An integrative review. Clinical Psychology Review, 29 (6), 560-572. doi: http://dx.doi.org/10.1016/j.cpr..06.

Chechko, N., Augustin, M., Zvyagintsev, M., Schneider, F., Habel, U., Kellermann, T. (2013). Brain circuitries involved in emotional interference task in major depression disorder. Journal of Affective Disorders, in press.

Cisler, J. M., \& Olatunji, B. O. (2012). Emotion regulation and anxiety disorders. Curr Psychiatry Rep, 14(3), 182-187. doi: 10.1007/s11920-012-0. 
Ehring, T., Tuschen-Caffier, B., Schnulle, J., Fischer,S., \& Gross, J. J. (2010). Emotion regulation and vulnerability to depression: spontaneous versusinstructed use of emotion suppression and reappraisal. Emotion, 10(4), 563-572. doi: 10.1037/a0019.

Gallassi, R, Morreale, R. A \& Amore, M,. (2006). Memory impairment in patients with lateonset major depression: the effect of antidepressant therapy, J AffectDisorders, 91, 243-450.

Garnefski N, Boon S, Kraaij V. (2002). Relationshipsbetween cognitive strategies of adolescents anddepressive symptomatology across different typesof life event. J Youth Adolesc; 32(6): 401-8.

Garnefski N, Teerds J, Kraaij V, Legerstee J,Kommer T.(2003). Cognitive emotionregulationstrategies and depressive symptoms: Differencesbetween males and females. Pers Individ; 25:603-11.

Ghomri Givi, Hossein; Bashrpour, Sajjad; Narimani, Mohammad (2008). Comparison of automated and controlled data processing in patients with major depressive disorder and normal people and the effect of antidepressants on these variables. Psychological Studies, Faculty of Educational Sciences and Psychology, Alzahra University, Period 4, Number 1, pp. 87-103.

Goldin, P. R., \& Gross, J. J. (2010). Effects of mindfulness-based stress re duction (MBSR) on emotion regulation in social anxiety disorder. Emotion, 10 (1), 83-91. doi: 10.1037/a001.

Gratz, K. L., \&Romer, L. (2004). Multidimensional Assessment of Emotion Regulation and Dysregulation: Development, Factor Structure, and Initial Validation of the Difficulties in Emotion Regulation Scale. Journal of psychopathology and behavioral assessment, 26 (1), $41-54$.

Hamar, A, (2003). Automatic and Effortful Information Processing in Unipolar Major Depression, Scandinavian Journal of Psychology, 44, 409-416.

Hasin D, Liu X, Nunes E, mac cloud s, samet S. Effects of major depression on remission and relapse of substance dependence. Arch Gen Psychiatry 2001; 59:375-80.

Joormann, J. Davanzato, c. (2010). Emotion regulation in depression; examines the role of cognitive processes. Journal of Cognition \& Emotion, 24(6); 913-939.

Kaplan and Sadook (2003). Summary of Psychiatry of Behavioral Sciences. (Translated by Nosratullah Pourafkari, 2003). Tabriz: Sohrab.

Khadayari Fard, Mohammad, Saghi, Khashayar, Abedini, Yasamin (2006). Application of Cognitive Behavioral Family Therapy with Chiropractic in the Treatment of Mental Disorders (Muscular Bones). Master's Thesis. University of Tehran. Faculty of Psychology and Educational Sciences.

Klingman, A. (2009ᄀ). Psychological education: studying adolescent's interests from their own perspective, Journal of Adolescence.

Kush FR, Fleming LM. (2000). An innovative approach to short-term group cognitive therapy in the combined treatment of anxiety and depression. Group Dynamics.; 4(11): 176-83.

O’Mahena, H. Fedock, G. Hensh aw, E. Himle, J. \& et al. (2012). Modifying CBT for Perinatal Depression: What Do Women Want? A Qualitative Study. Cognitive and Behavioral Practice; 19(2):359- 371.

Oei T, Dingle G. (2007). The Effectiveness of group cognitive behavioral therapy for unipolar depressive disorder. J Affect Disord, ᄀ107(1-3): 5-21. 
Robertson D. (2010). The Philosophy of Cognitive - Behavioral Therapy: Stoicism as Rational and Cognitive Psychotherapy. London, karnac. PP 176-90.

Sadook, Benyamin James, Sadook, Virginia Alcote (2011). Summary of Psychiatry of Behavioral Sciences / Clinical Psychiatry, translated by Farzin Rezaei, Tehran: Arjmand

Sadook, Benyamin James; Sadook, Virginia (2007). Summary of Psychiatry Volume II (Farzin Rezaee, 2008), Tehran, Arjmand.

Saleh, Jabbar, Mahmoudi, Osman, Paydar, Masoud (2015). The Effectiveness of CognitiveBehavioral Therapy on Reducing Depression in Schoolchildren, Journal of Psychological Development of the Child - Volume II, No. 1-83-88.

Sara.L.d,Rosemarie, A., martin, D.,Rohsenow, J.(2008). Self-eficacy for cocaine abstinence: pretreatment correlates and relationship to outcomes. Journal Addict Behaviors,33(4),675688.

Shamloo, Sa'id (1985). Psychopathology. Tehran: Chahar.

Solati Dehkordi, Kamal; Mobasheri, Mahmoud (2012). The Effectiveness of CognitiveBehavioral Therapy alone and in combination with Family Mental Education on the Improvement of Signs of Major Depressive Disorder in Female Patients, Journal of Kerman University of Medical Sciences. 19 (5) .490-501.

Thomas LJ, Abel A, Ridgway N, Peters T, Kessler D, Hollinghurst S, et al.(2012). Cognitive behavioural therapy as an adjunct to pharmacotherapy for treatment resistant depression in primary care: The CoBalT randomized controlled trial protocol. Contemp Clin Trials; 33(2): 312-9.

Watkins, P,C.(2000).Martin, C. K \& Stern, L. D. Unconscious memory bias indepression: Perceptual and conceptual processes, Journal of AbnormalPsychology, 109, 282-289.

Weingartner, H, Eckardt, M, Grafman, J, Molchan, S, Putnam, K, Rawlings, R \& Sunderland, T. (1993). The Effects of Repetition on Memory Performance in Cognitively Impaired Patients, Neuropsychology, 7, 385-395.

Zare Bahram Abadi, Mehdi; Tayebeh Nazari (2014). Effectiveness of cognitive therapy group on depression and self-efficacy of family headed women, Quarterly Journal of Clinical Psychology. 14 (4). 83-97. 